DOI:

10.1038/nrd2366

DRUG DELIVERY

\title{
Minicells deliver lethal load to tumours
}

\begin{abstract}
A key obstacle in the use of chemotherapeutic anticancer drugs is their lack of specificity for cancer cells, resulting in severe toxicity when they are administered systemically. A potential strategy to limit toxicity is to encapsulate the drug and target it directly to cancer cells. However, current strategies that use liposomes, nanoparticles or polymer therapeutics are hampered by shortcomings such as drug leakage or difficulties in scaling-up production. Reporting in Cancer Cell, MacDiarmid and colleagues now describe a promising new technology that firmly encapsulates chemotherapeutics into bacterial minicells, which are then guided by antibodies to deliver their lethal load to cancer cells.
\end{abstract}

The unusual drug delivery vehicle was generated by inactivating genes that control normal cell division in bacteria. This led to the formation of anucleate particles that have a uniform diameter of $400 \mathrm{~nm}$, and high yields are readily produced from both Gram-positive and Gram-negative bacteria. Purification to remove parental bacteria, membrane blebs, nucleic acids, cellular debris and free endotoxin resulted in sterile minicell preparations that could be lyophilized and stored for at least 4 months.

The minicells were loaded with therapeutically significant concentrations of chemotherapeutics of differing charge, hydrophobicity and solubility by simple incubation. Drug uptake occurred by diffusion - a process that fortunately proved to be unidirectional - and no drug leakage could be detected. Cancer-cell targeting was achieved by coupling minicells to bispecific antibodies, in which one arm recognizes surface lipopolysaccharide (LPS), and the other a surface receptor on the targeted cell, such as epidermal growth factor receptor (EGFR), HER2/neu (also known as ERBB2), CD33 or CD3. Interestingly, compared to 'armed antibodies', which can be conjugated to 4-10 drug molecules, minicells can be packaged with 1-10 million drug molecules. The adhesion of the minicells to cancer cells was shown to trigger receptor-mediated endocytosis, with liberation of the drug into the cytosol and nucleus of the targeted cell.

In vivo experiments with targeted doxorubicin-loaded minicells led to the dramatic inhibition and regression of tumour growth in mice that had human breast, ovarian, leukaemia or lung cancer xenografts. Importantly, comparatively small amounts of drug were required to induce tumour regression $-100 \mathrm{x}$ higher doses were required with liposome-encapsulated doxorubicin, and even $1,875 \mathrm{x}$ the amount of free doxorubicin did not produce comparable xenograft growth inhibition. For the hydrophobic drug paclitaxel, 8,000-fold higher doses of free drug were required for comparative results to the minicell-packaged drug. Furthermore, the drug was undetectable in the plasma of minicell-treated animals, and none of the animals developed any signs of toxicity.

The anticancer efficacy of the minicells was further evaluated in dogs with advanced T-cell non Hodgkin's lymphoma; treatment led

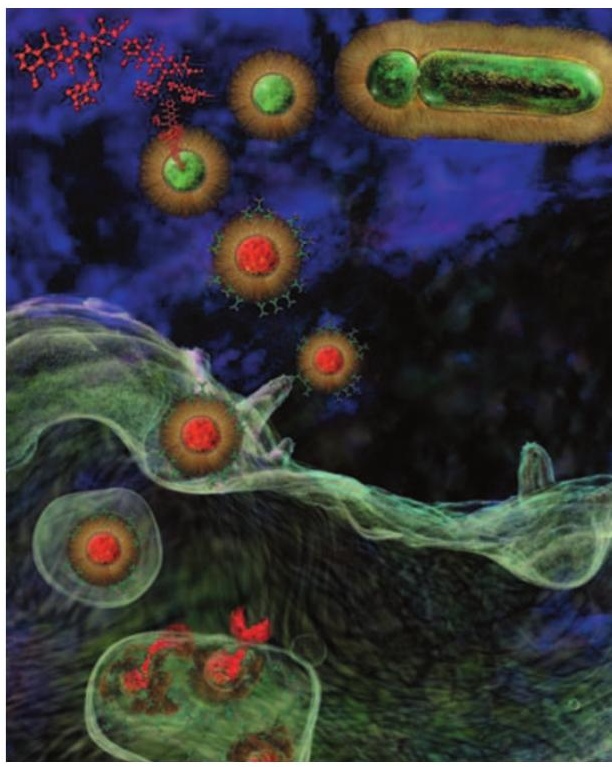

Courtesy of Russell Kightley Media, Canberra, Australia

to marked tumour regression and tumour lysis. Importantly, the treatment was tolerated without adverse side effects despite repeat dosing, and there was no increase in pro-inflammatory cytokines. Further experiments in pigs confirmed no adverse reactions in terms of haematological indices, serum chemistries, food intake or growth, and surprisingly anti-LPS titres remained at background levels.

Although the authors caution that extensive toxicology and stability studies would be required for the use of bacterial minicells in humans, these results might form the basis of a robust and versatile drug carrier system. Furthermore, the ability to administer very small, yet targeted doses of chemotherapeutics could allow the therapeutic potential of highly toxic agents to be realized.

Alexandra Flemming

ORIGINAL RESEARCH PAPER MacDiarmid, J. A.

et al. Bacterially derived $400 \mathrm{~nm}$ particles for

encapsulation and cancer cell targeting of chemotherapeutics. Cancer Cell 11, 431-445 (2007) 